\title{
Tailoring molecular island shapes: Influence of microscopic interaction on mesostructure
}

\author{
Simon Aeschlimann ${ }^{1,2}$, Lu Lyu $^{3}$, Benjamin Stadtmüller ${ }^{3,4}$, Martin Aeschlimann $^{3,4}$, and Angelika Kühnle ${ }^{5}(\bowtie)$ \\ ${ }^{1}$ Institute of Physical Chemistry, Johannes Gutenberg University Mainz, Duesbergweg 10-14, Mainz 55099, Germany \\ ${ }^{2}$ Graduate School Materials Science in Mainz, Staudingerweg 9, Mainz 55128, Germany \\ ${ }^{3}$ Department of Physics and Research Center OPTIMAS, University of Kaiserslautern, Erwin-Schrödinger-Straße 46, Kaiserslautern 67663, \\ Germany \\ ${ }^{4}$ Graduate School Materials Science in Mainz, Erwin-Schrödinger-Straße 46, Kaiserslautern 67663, Germany \\ ${ }^{5}$ Physical Chemistry I, Faculty of Chemistry, Bielefeld University, Universitätsstraße 25, Bielefeld 33615, Germany
}

(C) The Author(s) 2020

Received: 15 November 2019 / Revised: 7 February 2020 / Accepted: 9 February 2020

\begin{abstract}
Controlling the structure formation of molecules on surfaces is fundamental for creating molecular nanostructures with tailored properties and functionalities and relies on tuning the subtle balance between intermolecular and molecule-surface interactions. So far, however, reliable rules of design are largely lacking, preventing the controlled fabrication of self-assembled functional structures on surfaces. In addition, while so far many studies focused on varying the molecular building blocks, the impact of systematically adjusting the underlying substrate has been less frequently addressed. Here, we elucidate the potential of tailoring the mesoscopic island shape by tuning the interactions at the molecular level. As a model system, we have selected the molecule dimolybdenum tetraacetate on three prototypical surfaces, $\mathrm{Cu}(111), \mathrm{Au}(111)$ and $\mathrm{CaF}_{2}(111)$. While providing the same hexagonal geometry, compared to $\mathrm{Cu}(111)$, the lattice constants of $\mathrm{Au}(111)$ and $\mathrm{CaF}_{2}(111)$ differ by a factor of 1.1 and 1.5 , respectively. Our high-resolution scanning probe microscopy images reveal molecular-level information on the resulting islands and elucidate the molecular-level design principles for the observed mesoscopic island shapes. Our study demonstrates the capability to tailor the mesoscopic island shape by exclusively tuning the substrate lattice constant, in spite of the very different electronic structure of the substrates involved. This work provides insights for developing general design strategies for controlling molecular mesostructures on surfaces.
\end{abstract}

\section{KEYWORDS}

scanning tunneling microscopy (STM), self-assembly, island shape, mesostructure, scanning probe microscopy (SPM), twodimensional structures

\section{Introduction}

One of the crucial challenges in the miniaturization of device structures for further information technology and photonic applications is to devise novel concepts to fabricate active functional units on surfaces with atomic or molecular precision at the nanoscale. In this context, molecular self-assembly processes on surfaces have emerged as a powerful tool for creating functional molecular nanostructures on surfaces [1-6]. Making use of the tremendous variability of the molecular building blocks, the intermolecular and the molecule-surface interactions can be controlled to arrive at a specific surface structure that is encoded in the involved constituents $[7,8]$. In many cases, the molecule-substrate interaction has been recognized to be governed by the electronic structure of the substrate, which, in turn, impacts the structural properties of self-assembled nanomaterials on various substrates; e.g., bulk metals [9-15], twodimensional materials $[16,17]$, and polar oxide surfaces [18-20] Tuning the subtle balance between molecule-molecule and molecule-surface interactions [21] has opened up new pathways for creating an impressive variety of molecular structures on surfaces [22-26]. For example, structures ranging from perfectly ordered two-dimensional layers [27], unidirectional rows [28, $29]$, porous networks $[30,31]$ to complex structures have been presented as a function of the specific molecular design [27, 32, 33]. Even richer structures have been demonstrated in multicomponent systems, i.e., upon co-deposition of two types of molecules [34-39].

Despite these impressive results, a reliable structure prediction and rational control of the resulting structures is still largely lacking. In particular, the focus of intense research has been on exploring the variability of the molecular building blocks [24], while the influence of systematically varying the underlying substrate is rarely addressed. This is unfortunate as the substrate can act as a template $[40,41]$, directing molecular structure formation and providing a further dimension in rationally controlling molecular self-assembly on surfaces [42]. Moreover, to successfully employ molecular self-assembly in nano-structuring processes, it is also of utmost importance to control the structure formation on a mesoscopic scale. Here, we focus on this aspect and show that the mesoscopic shape of self-assembled molecular islands can be controlled by tuning the involved interactions 
at the molecular scale. In particular, we demonstrate that the shape of molecular islands can be tuned by adjusting the lattice constant of the underlying surface.

In the bulk of a molecular crystal, the molecular order is solely determined by the intermolecular interactions. In contrast, adsorbed on a surface, the spacing between neighboring molecules within an island can be tuned by using different substrates with different lattice constants due to the additional molecule-substrate interaction. For this surface templating effect, a sufficiently high and site-specific molecule-substrate interaction is necessary. Otherwise, the shape of the molecular island cannot be influenced in a controlled manner by the substrate periodicity.

To be more specific, as illustrated in Fig. 1(a), molecules on a surface can interact with the underlying substrate (I) and neighboring molecules (II). In a simple first approximation, the interactions governing molecular structure formation on surfaces may be considered to be independent of each other. In general, adsorbates are not rotational invariant, which can lead to anisotropic interactions. Thus, the intermolecular interaction can strongly depend on the direction as demonstrated in Fig. 1(a), showing an example for an island stabilized by two different intermolecular interactions (IIa and IIb).

The resulting mesoscopic island shape reflects the mismatch of the bulk crystal unit cell dimensions and the surface lattice constant. Even though, in this simple approximation, the intermolecular interaction is assumed to be independent of the strength of the molecule-substrate interaction, it remains being a function of the molecule-molecule distance. If the surface lattice constant equals to an integer multiple of the molecular crystal unit cell dimensions, the molecules can adopt their ideal spacing along this direction. As a consequence, an island elongated along this direction is thermodynamically favorable. This is why the thermodynamically most stable mesoscopic island shape is a direct consequence of the subtle balance of the involved direction-dependent intermolecular interactions and the molecule-surface interaction. This provides the opportunity to rationally design the molecular island shape by tuning the lattice constant of the underlying crystal.

In this work, we explore this particular opportunity of tuning the mesoscopic island shape by acting on the microscopic interactions in the system at the molecular scale. An ideal molecule for this purpose is the binuclear neutral complex dimolybdenum tetraacetate $\left(\mathrm{Mo}_{2}\left(\mathrm{O}_{2} \mathrm{CMe}\right)_{4}\right.$; in the following referred to as MoMo) shown in Fig. 1(b). Despite its rotational symmetry, adsorbed on a surface, MoMo exhibits a strong anisotropy in the intermolecular interactions as illustrated in Fig. 1(a).

As model substrates, we have selected three hexagonal surfaces $\mathrm{Cu}(111), \mathrm{Au}(111)$ and $\mathrm{CaF}_{2}(111)$ with different lattice constants. The lattice constant of $\mathrm{Au}(111)$ is slightly larger (1.1 times) and the lattice constant of $\mathrm{CaF}_{2}(111)$ is clearly larger (1.5 times) than the one of $\mathrm{Cu}(111)$. All surfaces form sufficiently strong, site-specific bonds to MoMo, making them highly suited for our surface templating study. Using scanning probe techniques, we study the self-assembly of submonolayer coverages of MoMo on these three surfaces. In all cases, the MoMo molecules adsorbed on these substrates form islands composed of molecules arranged in chains with a superstructure that is commensurate to the underlying lattice. Due to the different surface lattice constants, the commensurability results in different molecule-molecule distances and, hence, different molecule-molecule interaction strengths. This change at the molecular level has a profound influence on the mesoscopic island shape.

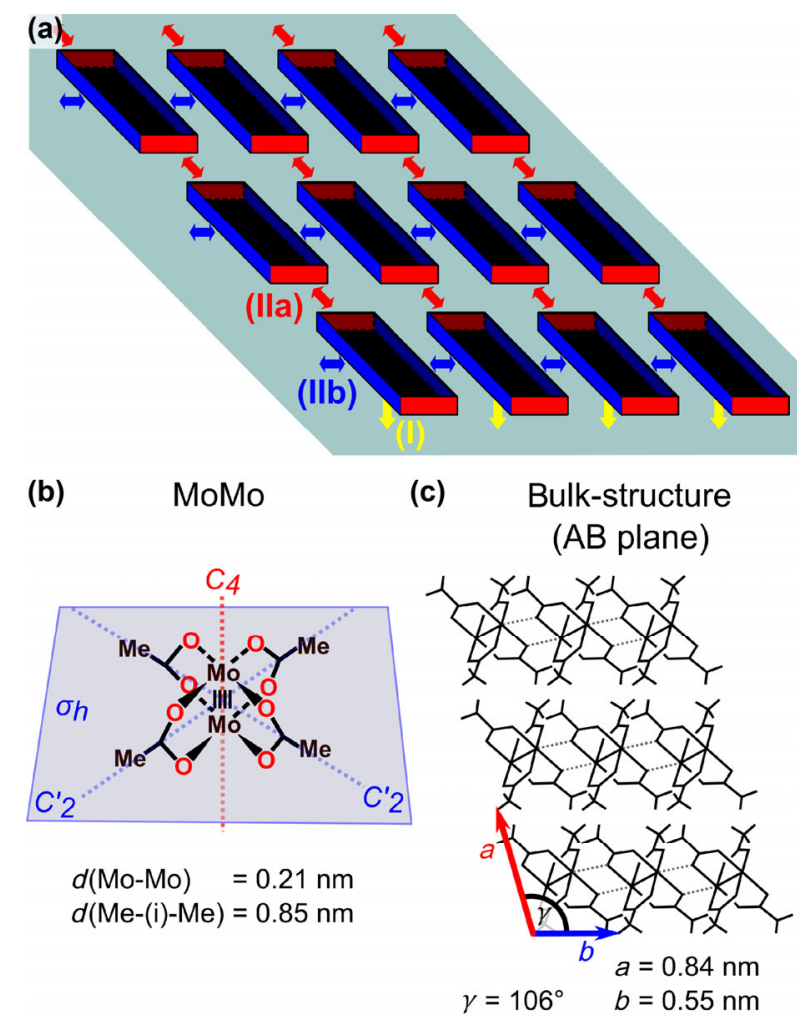

(d) $\mathrm{Cu}(111) \quad \mathrm{Au}(111) \quad$ (e) $\quad \mathrm{CaF}_{2}(111)$

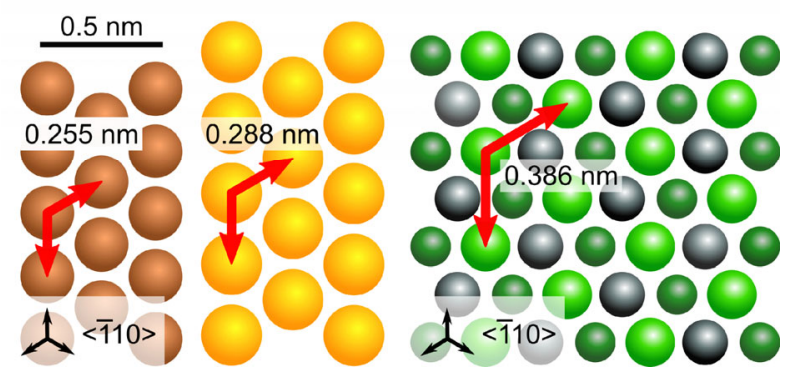

Figure 1 (a) Sketch of anisotropic interactions in a molecular island adsorbed on a surface. The yellow arrows indicate the molecule-substrate interaction (I). The two-fold rotational symmetry of the molecules results in two different intermolecular interactions marked with a blue arrow (IIa) and a red arrow (IIb). In a simple first approximation, all interactions are considered to be independent of each other. Changing the molecule spacing along one direction will only affect the strength of the corresponding interaction and, thereby, change the subtle balance between the intermolecular interactions (IIa and IIb). Model of (b) the MoMo molecule and (c) top view of its crystal AB-plane consisting of stacked molecular chains. The MoMo molecules interact electrostatically between the positively charged molybdenum atoms and the oxygen atoms of the neighboring molecules, indicated by the grey dotted line. (d) Model of the hexagonal copper/gold (111) structure with a lattice constant of $a_{\mathrm{Cu}}=0.255 \mathrm{~nm}$ and $a_{\mathrm{Au}}=0.288 \mathrm{~nm}$, respectively. (e) Model of the calcium fluoride (111) surface. Both, fluorine (green) and calcium (grey) ions are arranged in a hexagonal manner with a periodicity of $a_{\mathrm{CaF}_{2}}=0.386 \mathrm{~nm}$, leading to a three-fold symmetry. Protruding fluorine ions are highlighted with a brighter green color. The $0.5 \mathrm{~nm}$ scale bar applies to (d) and (e).

Our work demonstrates that, in spite of the distinctly different nature of these surfaces (ionic surface versus noble metal surface), the driving force determining the island shape can be sufficiently described by a simple comparison of the superstructure unit cell dimensions and the molecular bulk structure. As long as site-specific adsorption is present, the driving force can even be reduced to the aspect of surface geometry (symmetry and periodicity). As a result, in this case, the microscopic electronic structure of the substrate does not 
need to be considered for explaining the mesoscopic structure. Therefore, our work illustrates a way to control the mesoscopic island shape of molecules on surfaces by tuning the substrate lattice constants.

\section{Materials and methods}

Dimolybdenum tetraacetate $\left(\mathrm{Mo}_{2}\left(\mathrm{O}_{2} \mathrm{CMe}\right)_{4}\right.$, in this work referred to as MoMo) is a binuclear neutral complex of the transition metal molybdenum. It belongs to the $\mathrm{D}_{4 \mathrm{~h}}$ symmetry point group (see Fig. 1(b)). Historically seen, MoMo is of unique significance, because it is a prime example for an intramolecular quadruple bond between two transition metals; and it generally serves as a precursor for the synthesis of other known compounds containing molybdenum quadruple bonds [43]. For the MoMo molecule, the quadruple bond manifests itself in an intramolecular Mo-Mo bonding distance of approximately $0.21 \mathrm{~nm}$ [44]. This is significantly shorter than the distance of $0.27 \mathrm{~nm}$ in a molybdenum single crystal [45].

The MoMo crystal has a triclinic superstructure as firstly reported by D. Lawton and R. Mason in 1965 [46]. Later, the triclinic crystal structure has been confirmed and corresponding lattice constants have been redetermined in higher accuracy in extensive diffraction measurements by F. A. Cotton et al. [47] and K. Hino et al. [48]. The molybdenum crystal AB plane, defined by the lattice constants $a$ and $b$, is characterized by upright standing molecules being arranged next to each other along the $b$-direction (Fig. 1(c)). In the following, we will refer to this arrangement as molecular chains. The chains are stabilized by an intermolecular interaction between a molybdenum atom and an oxygen atom of the neighboring molecule (marked by a grey, dotted line in Fig. 1(c)). In the crystal structure, the molecules are slightly tilted with respect to the chain direction in order to optimize the configuration of the metal-metal axial position for the bridging Mo-O interaction [49]. Ultraviolet photoelectron spectroscopy (UPS) measurements indicate a $\sigma-\pi$ orbital splitting, presumably caused by this axial intermolecular interaction [50]. The lattice constant, i.e., the periodicity of the MoMo molecules along the chains in the bulk structure is $b=0.550 \mathrm{~nm}^{1}$ (indicated by a blue arrow in Fig. 1(c)). In the $\mathrm{AB}$ plane of the MoMo crystal, the molecular chains are stacked up next to each other. This leads to a lattice constant of $a=0.842 \mathrm{~nm}$ (indicated by a red arrow in Fig. 1(c)), which represents the distance of two molecules in neighboring chains. In the following, we will refer to the stacking direction $a$ (red arrows) and the chain direction $b$ (blue arrows). In the bulk structure, the two directions are enclosing an angle of $\gamma=106^{\circ}$. (2)

The (111) surfaces of the metallic $\mathrm{Cu}$ and $\mathrm{Au}$ crystals exhibit a hexagonal surface structure (see Fig. 1(d)). The $\mathrm{CaF}_{2}$ crystal is a bulk insulator consisting of fluorine and calcium triple layers stacked in the order of $\left(\mathrm{F}^{-}-\mathrm{Ca}^{2+}-\mathrm{F}^{-}\right)_{n}$ along the [111] direction. Even so the layers are charged, there exists no net dipole moment perpendicular to the surface [51]. Figure 1(e) illustrates the $\mathrm{CaF}_{2}(111)$ surface. The positively charged $\mathrm{Ca}^{2+}$ ions (grey) and the negatively charged flour $\mathrm{F}^{-}$ions (green) are arranged in a hexagonal manner with a surface lattice constant of $a_{\mathrm{CaF}_{2}}=0.386 \mathrm{~nm}$. Thus, all substrates studied in this work, $\mathrm{Cu}(111), \mathrm{Au}(111)$ and $\mathrm{CaF}_{2}(111)$, possess a three-fold rotational symmetry. However, the lattice constant of $\mathrm{Cu}(111)$ is $a_{\mathrm{Cu}}=$ $0.255 \mathrm{~nm}$ and, hence, slightly smaller compared to $a_{\mathrm{Au}}=$

(1) Following the notation of D. Lawton et al. (1965).

(2) Please note, the lattice constant $a$ along the stacking direction is not the distance between two chains, because this distance would be measured perpendicular to the chains.
$0.288 \mathrm{~nm}$, and roughly 1.5 times smaller compared to the insulator. Thus, $\mathrm{Cu}(111), \mathrm{Au}(111)$ and $\mathrm{CaF}_{2}(111)$ share the same hexagonal lattice, but exhibit different lattice constants. Therefore, these substrates constitute an ideal model system to examine how the molecular island shape is affected by tuning the substrate lattice.

All experiments shown in this work were carried out with scanning probe microscopes from Scienta Omicron (VT AFM $\mathrm{XA}$ ) under ultrahigh vacuum (UHV) conditions. Dynamic AFM (atomic force microscopy) experiments of MoMo on calcium fluoride were performed in frequency modulation mode [52]. We used n-doped silicon cantilevers from Nanosensors with a nominal force constant of $40 \mathrm{~N} \cdot \mathrm{m}^{-1}$ and an eigenfrequency of $300 \mathrm{kHz}$ in UHV. For the scanning tunneling microscopy (STM) measurements on $\mathrm{Cu}(111)$ and $\mathrm{Au}(111)$, another system from Scienta Omicron (VT AFM XA) was operated in constant current mode with the tunneling current $I_{t}$ usually in the range of 70-90 pA. Positive and negative values correspond to tunneling from the occupied and into the unoccupied states of the sample, respectively.

The preparation of the bare surfaces as well as the molecule deposition were performed in situ with a chamber base pressure typically better than $10^{-10}$ mbar. Optical quality calcium fluoride $\left(\mathrm{CaF}_{2}\right)$ crystals were purchased from Korth Kristalle $\mathrm{GmbH}$, Kiel, Germany. Prior to molecule sublimation, the $\mathrm{CaF}_{2}$ crystal was annealed to $400 \mathrm{~K}$ for $1 \mathrm{~h}$. The $\mathrm{Cu}(111)$ and $\mathrm{Au}(111)$ single crystals from MaTeck, Jülich, Gemany were cleaned by repeated cycles of $\mathrm{Ar}^{+}$sputtering (1.5 keV, $20 \mathrm{~min}$ ) and a subsequent annealing to about $850 \mathrm{~K}$. The cleanliness and the surface morphology were inspected by recording large-scale STM images and low-energy electron diffraction (LEED).

The MoMo molecules were bought from Sigma-Aldrich, Germany, with a purity of $98 \%$, and the powder was further purified in advance by long-time out-gassing at $400 \mathrm{~K}$ in a UHV molecular beam evaporator system. Subsequently, MoMo molecules were deposited using a home-built Knudsen cell that is heated for molecule sublimation. For the experiments shown here, molecules were sublimated for 10-50 min at a typical temperature of $440 \mathrm{~K}$ onto the sample at a distance of approximately $9 \mathrm{~cm}$.

\section{Results}

In the first part of the result section, STM measurements of submonolayers of MoMo on $\mathrm{Cu}(111)$ are presented, which revealed a nearly perfect fit to the bulk periodicity along the chain direction. The self-assembly of this system has first been reported by Kollamana et al. [53]. Here, we therefore only summarize the most important conclusions concerning the molecule adsorption position. In addition, new, large overview STM images were taken in the present study to determine the island mesoscopic structure.

After submonolayer deposition of MoMo on $\mathrm{Cu}(111)$ at about $110 \mathrm{~K}$, the co-existence of two different island structures is revealed (see Fig. 2(a)). Beside a chain phase (marked with blue lines), being similar to the $\mathrm{AB}$ plane of the molecular bulk, a second structure referred to as mesh phase (marked in cyan) is present [53]. The co-existence of the structures suggests that both structures are energetically similar. Here, the mesh phase, which is formed by flat-lying molecules, is not considered any further, because in this work we will focus on the chain phase solely, which is composed of upright-standing molecules.

An overview STM image (Fig. 2(a)) indicates that the islands of the chain phase on $\mathrm{Cu}(111)$ are elongated along the chain 


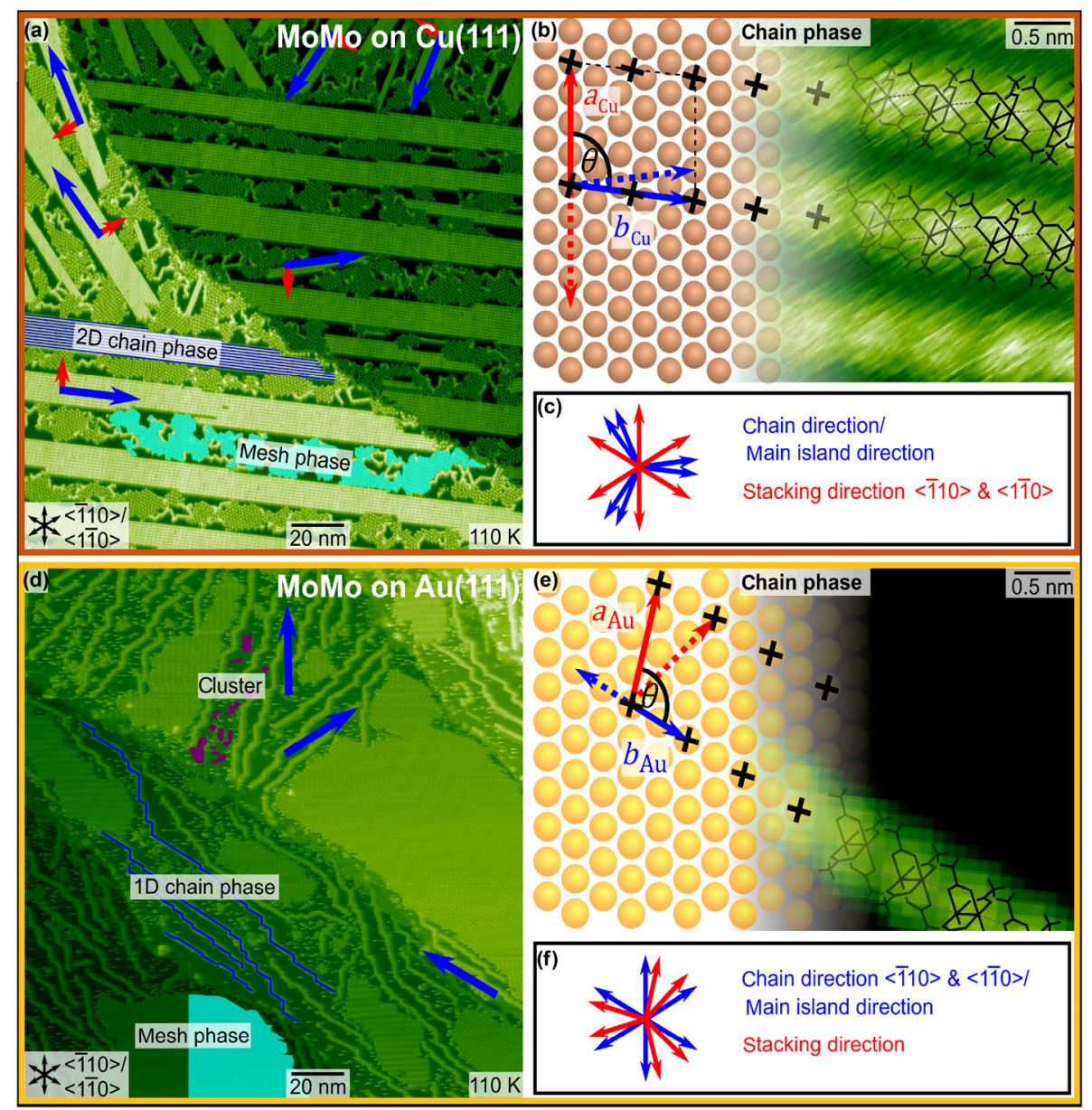

Figure 2 (a) and (d) STM image of MoMo on $\mathrm{Cu}(111)$ and $\mathrm{Au}(111)$ at a coverage of $\theta=0.70 \mathrm{ML}$ at about $110 \mathrm{~K}$. For illustration, one $2 \mathrm{D}$ chain phase island on $\mathrm{Cu}(111)$ and a few $1 \mathrm{D}$ chain phase islands on $\mathrm{Au}(111)$ are exemplary marked with dark blue lines. In both images part of a mesh phase island is marked in cyan. On $\mathrm{Au}(111)$, some molecule clusters are exemplary marked in purple. Blue arrows indicate main island growth directions of the chain phase. (b) and (e) Schematic representation of the chain phase superstructure superimposed onto a high-resolution STM image. The black crosses mark possible molecule adsorption position with respect to the $\mathrm{Cu}(111) / \mathrm{Au}(111)$ lattice. The unit cell parameters on $\mathrm{Cu}(111) \mathrm{are} a_{\mathrm{Cu}}=1.020 \mathrm{~nm}$, $b_{\mathrm{Cu}}=1.110 \mathrm{~nm}$ and $\theta_{\mathrm{Cu}}=96.6^{\circ}$ [53]. On $\mathrm{Au}(111)$ the islands are only elongated along the chain direction. However, a virtual unit cell is given by $a_{\mathrm{Au}}=1.036 \mathrm{~nm}, b_{\mathrm{Au}}=0.576 \mathrm{~nm}$ and $\theta_{\mathrm{Au}}=106^{\circ}$. A mirrored island structure is illustrated with dotted arrows. (c) and (f) The observed molecule stacking directions are indicated with red arrows and the chain directions are indicated with blue arrows. For both surfaces, there exist six energetically equal, but different oriented island structures. For $\mathrm{Cu}(111)$, the stacking directions are oriented along the $\langle\overline{1} 10\rangle$ and $\langle 1 \overline{1} 0\rangle$ directions and for $\mathrm{Au}(111)$ the chain directions.

directions (blue arrows) and are rather limited in the stacking direction (red arrows). The islands can be over $300 \mathrm{~nm}$ long along the chain direction. The chain length is limited by a step edge or another chain phase island. In contrast to that, the islands width seems to be limited to roughly $30 \mathrm{~nm}$, consisting of about 30 chains. In total six different chain directions (indicated by blue arrows) have been observed [53].

Figure 2(b) shows an illustration of MoMo adsorbed on the $\mathrm{Cu}$ (111) surface, superimposed onto a zoom image of the chain phase. The exact adsorption position on the surface is unknown, but also irrelevant for the following discussion. However, the black crosses in Fig. 2(a) indicate the relevant periodicity of the superstructure determined by STM. The unit cell along the chain direction is marked with a blue arrow $\left(b_{\mathrm{Cu}}=1.110 \mathrm{~nm}\right)$ and along the stacking direction with a red arrow $\left(a_{\mathrm{Cu}}=1.020 \mathrm{~nm}\right)$, enclosing an angle of $\theta=96.6^{\circ}$. This adsorbates unit cell is commensurate to the underlying substrate. Each unit cell contains two molecules, with two different molecule adsorption positions within the unit cell. The molecules are separated by 0.530 and $0.580 \mathrm{~nm}$ along the chain direction [53]. Hence, on $\mathrm{Cu}(111)$ a second, probably energetically less favorable, adsorption position is accepted to enable a nearly optimal molecule-molecule distance along the chain direction.

All stacking directions point along the high-symmetry $\langle\overline{1} 10\rangle$ and $\langle 1 \overline{1} 0\rangle$ surface directions. For each stacking direction, one chain direction is favorable. The two opposite directions, $\langle\overline{1} 10\rangle$ and $\langle 1 \overline{1} 0\rangle$, therefore result in two mirrored island structures. In Fig. 2(b), the mirrored adsorption structure is marked with the dotted arrows. As expected from the surface symmetry, on $\mathrm{Cu}(111)$ this leads in total to six energetically equivalent island structures (see Fig. 2(c)), composed of six different island stacking directions (indicated by red arrows) and six different chain directions (indicated with blue arrows).

With the experiments presented here, we cannot determine the absolute microscopic adsorption position. However, the unit cell of the molecular adsorbates can be measured precisely, which is the key indicator for predicting the shape of the molecular islands at the mesoscale. With this knowledge, we now tune the mesoscopic molecular island shape by changing the underlying lattice periodicity. Upon increasing the lattice constant by changing from $\mathrm{Cu}(111)$ to $\mathrm{Au}(111)$, we induce a slightly larger molecule distance along the stacking direction (red) as compared to on $\mathrm{Cu}(111)$. Therefore, the lattice constant on $\mathrm{Au}(111)$ constitutes a nearly perfect fit to the bulk periodicity along the chain direction. 
Figure 2(d) shows an overview STM image of MoMo on $\mathrm{Au}(111)$. The image again reveals a coexistence of two different island types. Based on a comparison with what we have found on $\mathrm{Cu}(111)$, we again find a phase of flat lying molecules (marked in cyan) and a phase that is composed of upright standing molecules (marked in blue). Therefore, we assign the structure of flat-lying molecules to the mesh phase and the structure of upright-standing molecules to the chain phase. In addition, some unordered molecular clusters are visible (marked in purple). For comparison, we again focus only on the chain phase. On $\mathrm{Au}(111)$, the chain phase consists solely of single, one-dimensional rows along the main symmetry directions $\langle\overline{1} 10\rangle$ and $\langle 1 \overline{1} 0\rangle$. On $\mathrm{Au}(111)$, a row length of up to about $200 \mathrm{~nm}$ is observed in the STM images. Similar to the two-dimensional chain phase on $\mathrm{Cu}(111)$, the one-dimensional chains on $\mathrm{Au}(111)$ end at step edge or other islands.

An illustration of MoMo adsorbed on the Au(111) surface, superimposed onto a zoom image of the chain phase is shown in Fig. 2(e). The chain phase islands solely form one-dimensional rows along the chain direction, extension along the stacking direction is not observed. In order to exclude a kinetically trapped structure, we have performed STM images at different temperatures in the range from $110 \mathrm{~K}$ up to $300 \mathrm{~K}$. Even at elevated temperature, no change in the island shape is observed. Therefore, the extension along the stacking direction must be energetically unfavorable. The periodic molecule distance along the chain direction is marked with a blue arrow and black crosses $\left(b_{\mathrm{Au}}=0.576 \mathrm{~nm}\right)$. Following the substrates periodicity, a virtual two-dimensional unit cell is spanned up with a red arrow $\left(a_{\mathrm{Cu}}=1.04 \mathrm{~nm}\right)$ along the stacking direction, enclosing an angle of $\theta=106^{\circ}$. This commensurable, virtual unit cell is identified by considering the next identical adsorption position upon keeping the unit cell dimension and angle as close to the molecular bulk unit cell as possible. However, such a hypothetical two-dimensional structure seems to be energetically unfavorable, because only single rows are observed. A mirrored adsorption structure is illustrated with dotted arrows. In total, six different chain directions along the $\langle\overline{1} 10\rangle$ and $\langle 1 \overline{1} 0\rangle$ directions are observed, leading to six corresponding virtual stacking directions as illustrated in Fig. 2(f).

In the third part of the result section, the self-assembly of submonolayers of MoMo on $\mathrm{CaF}_{2}(111)$ is discussed. Prior to molecule sublimation, high-resolution AFM images of the freshly cleaved $\mathrm{CaF}_{2}$ crystal (see Fig. 3(a)) are performed in order to determine the crystals orientation. Depending on the tip termination, different contrasts have been observed for $\mathrm{CaF}_{2}(111)$ with either the $\mathrm{Ca}^{2+}$ or $\mathrm{F}^{-}$ions appearing brighter in the image $[54,55]$. Therefore, the grey circles in Fig. 3(a) indicate the substrate periodicity and do not indicate specific ion positions. The high-symmetry directions $\langle\overline{1} 10\rangle$ and $\langle 1 \overline{1} 0\rangle$, and the lattice vectors are marked with blue arrows in Fig. 3(a).

At $130 \mathrm{~K}$, dynamic AFM measurements are performed to determine the island structure as a function of the coverage (see Figs. 3(b)-3(d)). At a low coverage of $15 \%$ of a monolayer $(\theta=0.15 \mathrm{ML})$ several islands, pointing in various directions, are visible. However, all islands exhibit a similar shape of lacerated rectangles, being elongated in specific directions (exemplary marked with a red arrow). We define the direction along the longest island side as the main axis and the island extension perpendicular to it as the island width. When increasing the coverage to half of a monolayer (see Fig. 3(c)) the number of islands per area and the island length along the main island axis direction increase, while the island width remains rather constant. A further increase of the coverage to nearly one monolayer (see Fig. 3(d)) leads to further elongation of the islands, but the island width still remains nearly unchanged. In total, six different directions for the main island axis are identified as illustrated with the red arrows in Fig. 3(d). The directions of the main island axes are independent of the temperature and coverage. The histograms in Fig. 3(e) display the average island width and length as a function of coverage. As mentioned above, a clear trend is visible. The island width remains rather constant, while the length increases with higher coverage.

In order to elucidate the inner island structure, highresolution images are performed as shown in Fig. 4(a). Every island is made up of molecular chains lined up next to each other, similar to the arrangement in the bulk (see Fig. 1(c)). As
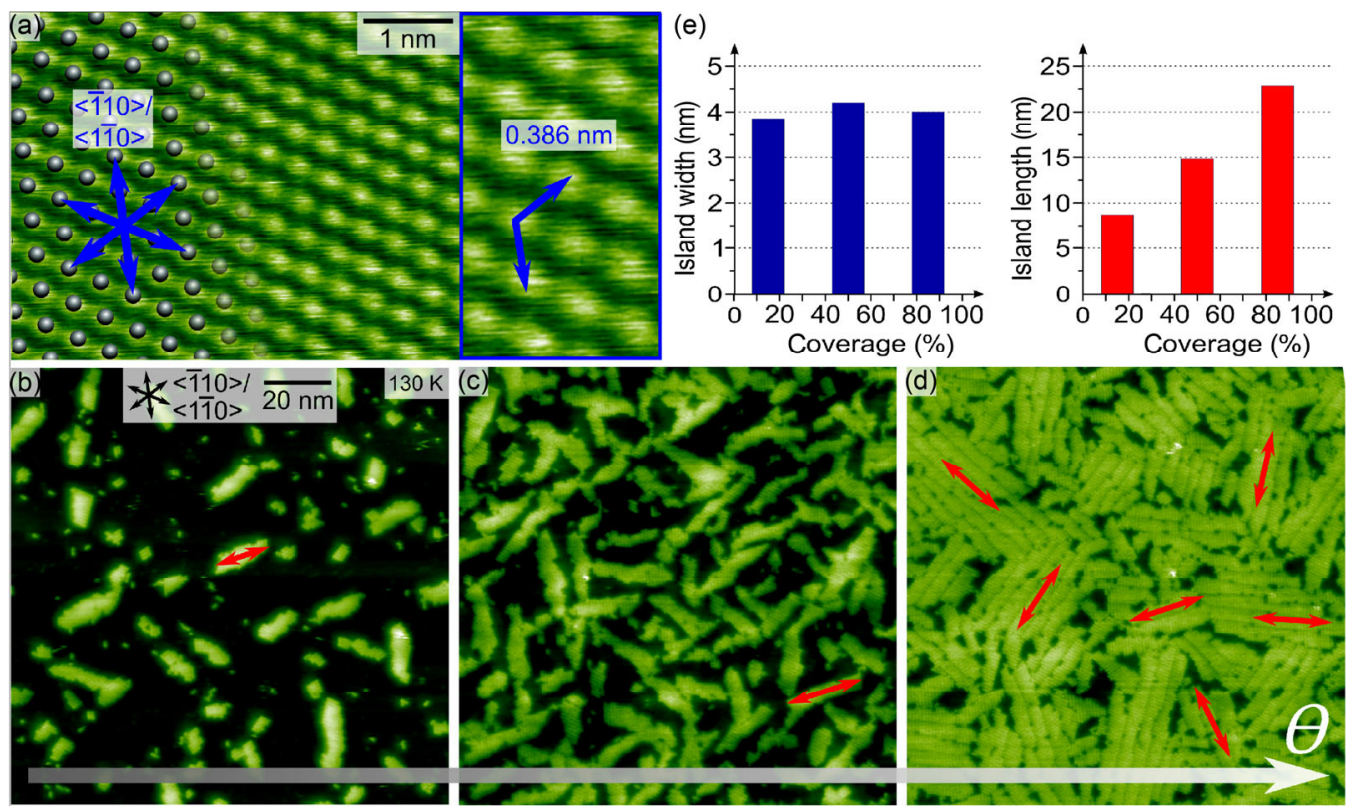

Figure 3 (a) AFM image of freshly cleaved $\mathrm{CaF}_{2}(111)$. High-symmetry directions $\langle\overline{1} 10\rangle$ and $\langle 1 \overline{1} 0\rangle$ are marked with blue arrows. The lattice constant is indicated in the zoom image. (b)-(d) AFM images of MoMo on $\mathrm{CaF}_{2}(111)$ as a function of molecule coverage (b) $\theta=0.15 \mathrm{ML}$, (c) $\theta=0.50 \mathrm{ML}$ and (d) $\theta=0.85 \mathrm{ML}$ at a fixed substrate temperature of about $130 \mathrm{~K}$. At high coverage, several islands with the same direction of the main island axis are arranged next to each other. Red arrows indicate six different directions for the island main axis. The scale bar in (b) also applies to the images (c) and (d). (e) Average island width and length as a function of coverage. The average width remains nearly constant, while the average length increases. 


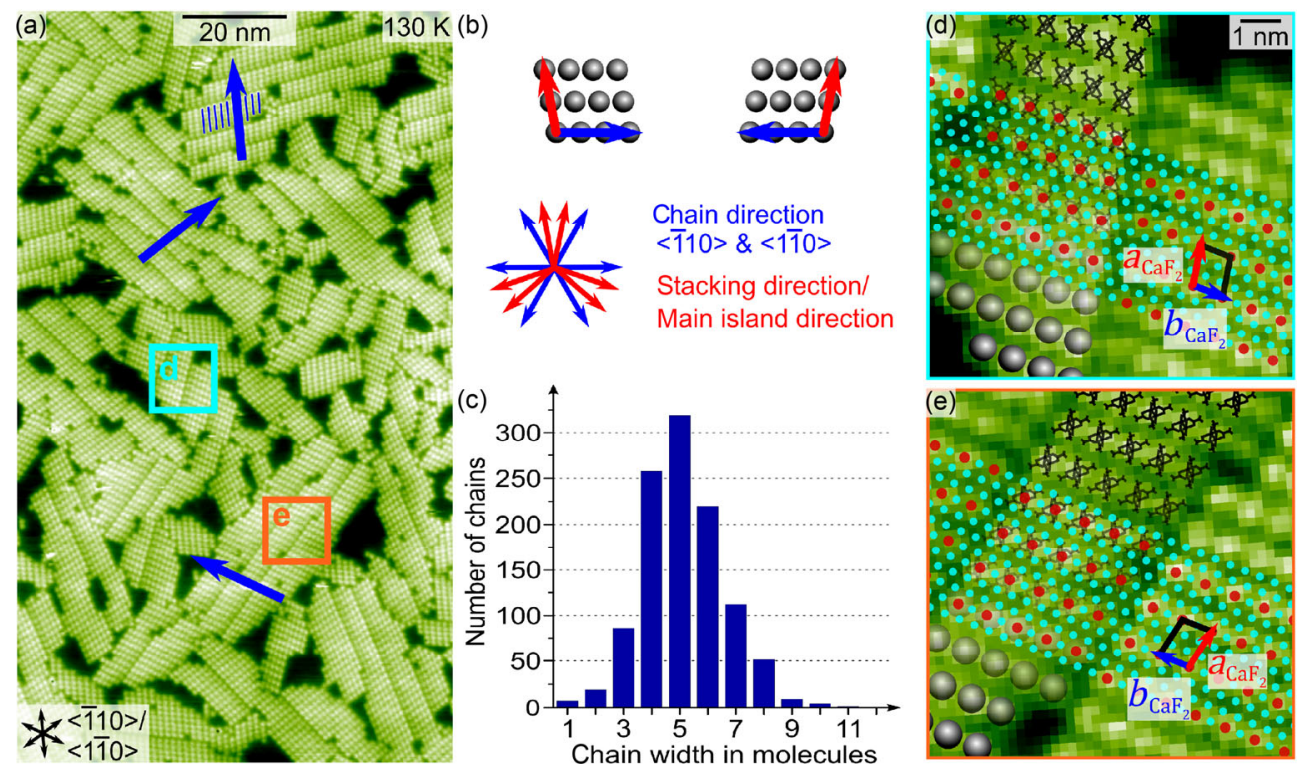

Figure 4 (a) High-resolution AFM image of $\mathrm{MoMo}_{\text {on }} \mathrm{CaF}_{2}$ (111) with an island coverage of $\theta=0.85 \mathrm{ML}$ at about $130 \mathrm{~K}$. Each island is made up of chains of molecules (indicated by blue arrows) oriented along the main surface symmetry directions $\langle\overline{1} 10\rangle$ and $\langle 1 \overline{1} 0\rangle$. (b) For each of the chain directions along $\langle\overline{1} 10\rangle$ a mirrored island structure with the chain direction along $\langle 1 \overline{1} 0\rangle$ exist (indicated with blue arrows), leading to six different directions for the main island axis (indicated with red arrows). (c) Histogram of frequency of counted chains in image (a) as a function of the chain width in molecules. The average island width is five molecules. (d) and (e) Zoom onto two island structures that are mirror images of each other. The cyan blue circles indicate the substrate periodicity. The red circles mark the molecule positions. The superstructure with a unit cell dimension of $b_{\mathrm{CaF}}^{*}=0.772 \mathrm{~nm}$ (blue arrow), $a_{\mathrm{CaF}_{2}}^{*}=1.021 \mathrm{~nm}$ (red arrow) and angle of $\gamma=100.9^{\circ}$ are marked with the red and blue arrow, respectively.

indicated with blue arrows in Fig. 4(a), the molecular chains follow the main surface symmetry directions $\langle\overline{1} 10\rangle$ and $\langle 1 \overline{1} 0\rangle$. For each chain direction along $\langle\overline{1} 10\rangle$ an energetically equivalent mirrored structure exists, with the chains pointing in the opposite $\langle 1 \overline{1} 0\rangle$ direction (see Fig. 4(b)). In total, this leads to six energetically equivalent island structures, composed of six chain directions (indicated with blue arrows) and one corresponding island stacking direction each (indicated by red arrows).

Quite contrary to the $\mathrm{Cu}(111)$ and $\mathrm{Au}(111)$ surface, the MoMo islands are mostly elongated (up to $50 \mathrm{~nm}$ ) along the six stacking directions and are rather short (around $4 \mathrm{~nm}$ ) in the six chain directions. Figure 4(c) displays a histogram of the chain widths frequency of occurrence. The histogram is obtained by counting the width of over 1,000 chains in the acquired AFM image shown in Fig. 4(a). The most frequent and also average chain width is five molecules. Approximately $7 \%$ of the chains have a width from four to six molecules and over $90 \%$ from three to seven molecules. For larger chain widths, the frequency of occurrence drastically decreases. The largest island width observed is eleven molecules. Therefore, a large island width must be energetically unfavorable. This might be a consequence of a slight offset of the molecule periodicity compared to the commensurability with the substrate.

Figures 4(d) and 4(e) show two mirrored MoMo islands on $\mathrm{CaF}_{2}(111)$ with the chain direction marked in blue and the stacking direction marked in red. Zooming onto the islands reveals the inner structure. The red circles highlight the molecule positions, which are commensurate with the periodicity of the substrate indicated by the cyan blue circles. ${ }^{1}$ The surface unit cell of the superstructure is marked with a parallelogram spanned by a blue vector $b_{\mathrm{CaF}_{2}}^{*}=0.772 \mathrm{~nm}$ along the chain direction and a red vector $a_{\mathrm{CaF}_{2}}^{z^{2}}=1.021$ along the stacking

(1) As described above, the periodicity and orientation of the substrate is known, but not the absolute ion position. Thus, the cyan circles only represent the periodicity and not the absolute position. direction enclosing an angle of $\gamma=100.9^{\circ}$. Inspired by the molecular bulk structure (see Fig. 1(c)), a proposed molecule geometry of nearly up-right standing molecules is superimposed on the islands in the image.

\section{Discussion}

In the following, the above described adsorption structure of submonolayers of MoMo on $\mathrm{Cu}(111), \mathrm{Au}(111)$ and $\mathrm{CaF}_{2}(111)$ are critically discussed and compared to the known bulk structure. This analysis reveals the impact of the substrate on the molecular self-assembly and elucidates how to take advantage of this knowledge to direct the molecules to automatically arrange in a desired mesoscopic island shape.

As described above, the $\mathrm{AB}$ plane of the bulk consists of molecular chains stabilized by an intermolecular interaction between the positively charged $(+1.17$ e) molybdenum atoms and the negatively charged $(-0.29$ e) oxygen atoms of the neighboring molecules [48]. In the bulk, this leads to an optimized molecule-molecule spacing of $0.550 \mathrm{~nm}$ along the chains (chain direction) and a distance of $0.842 \mathrm{~nm}$ between the chains, referred to as stacking direction (see Table 1).

First, we will compare and elucidate the mesoscopic island structure between the two similar metallic substrates $\mathrm{Cu}(111)$ and $\mathrm{Au}(111)$. In a second step, we will discuss in what manner the here described method of tuning the lattice constant can be applied to bulk insulators like $\mathrm{CaF}_{2}(111)$, which exhibit a completely different electronic structure.

Our study reveals that similar chain phase islands form after deposition of MoMo molecules on both, $\mathrm{Cu}(111)$ and on $\mathrm{Au}(111)$ [53]. On both surfaces, the molecule-substrate interaction leads to a commensurate superstructure.

For $\mathrm{Cu}(111)$, the lattice constant of $0.255 \mathrm{~nm}$ and its multiples do not fit well to the periodicity of $0.550 \mathrm{~nm}$ along the chain direction found in the MoMo bulk structure. However, on $\mathrm{Cu}(111)$ two different adsorption sites are occupied within one unit cell, resulting in a nearly ideal molecule-molecule distance of 0.530 and $0.580 \mathrm{~nm}$ (see Table 1). Consequently, 
Table 1 Unit cell dimensions for the bulk $A B$ plane and for the chain phase on $\mathrm{Cu}(111), \mathrm{Au}(111)$ and $\mathrm{CaF}_{2}(111)$. Lattice constants similar to the bulk structure are marked in green $(<10 \%)$ and major deviating lattice constants are marked in red. The deviation relative to the corresponding bulk lattice is given in percentage

\begin{tabular}{ccccc}
\hline MoMo $\ldots$ & $\begin{array}{c}\text { In bulk } \\
\text { structure }\end{array}$ & $\begin{array}{c}\mathrm{On} \mathrm{Cu} \\
(111)\end{array}$ & $\begin{array}{c}\mathrm{On} \mathrm{Au} \\
(111)\end{array}$ & $\begin{array}{c}\mathrm{On} \mathrm{CaF}_{2} \\
(111)\end{array}$ \\
\hline $\begin{array}{c}\text { Periodicity } b \\
\text { along chain } \\
\text { direction in nm }\end{array}$ & 0.550 & $\begin{array}{c}0.530 / 0.580 \\
(+5 \%)\end{array}$ & $\begin{array}{c}0.576 \\
(+5 \%)\end{array}$ & $\begin{array}{c}0.772 \\
(+40 \%)\end{array}$ \\
$\begin{array}{c}\text { Periodicity } a \\
\text { along stacking } \\
\text { direction in nm }\end{array}$ & 0.842 & $\begin{array}{c}1.020 \\
(+21 \%)\end{array}$ & $\begin{array}{c}1.036 \\
(+23 \%)\end{array}$ & $\begin{array}{c}1.021 \\
(+21 \%)\end{array}$ \\
Angle $\boldsymbol{\gamma}$ in & 106 & $\begin{array}{c}96 \\
(-9 \%)\end{array}$ & $\begin{array}{c}106 \\
( \pm 0 \%)\end{array}$ & $\begin{array}{c}101 \\
(-5 \%)\end{array}$ \\
\hline
\end{tabular}

along the chain direction MoMo prefers to have a nearly ideal molecule-molecule distance on the cost of a second, maybe energetically less favorable, adsorption position. However, along the stacking direction the molecule-molecule distance of $1.02 \mathrm{~nm}$ is approximately $20 \%$ larger than the ideal configuration in the MoMo bulk crystal. Presumably, the increased distance along the stacking direction weakens the interaction between neighboring chains, while the interaction of the molecules in the chains remains similar as in the bulk. Therefore, the substrate-induced change in the intermolecular interactions offers a straightforward explanation as to why the resulting island shape is elongated in the chain direction, as the intermolecularinteractions are stronger along this direction. Our large-scale STM measurements confirm this assumption (see Figs. 4 and 5): $\mathrm{On} \mathrm{Cu}$ (111), the chain phase islands are strongly elongated along the chain direction (indicated by a blue arrow) for more than $300 \mathrm{~nm}$, but much shorter in the stacking direction (indicated by a red arrow).

In contrast to the structure on $\mathrm{Cu}(111)$, a different overall island shape is found for the self-assembly of MoMo on $\mathrm{Au}(111)$. The lattice constant of $\mathrm{Au}(111)$ is $0.288 \mathrm{~nm}$, and hence, slightly larger than of $\mathrm{Cu}(111)$. Along the chain direction, two times the lattice constants of $\mathrm{Au}(111)$ results in a moleculemolecule distance of $0.576 \mathrm{~nm}$ (see Table 1), which already fits well to the ideal configuration in the bulk; allowing a strong molecule-molecule interaction along the chain direction. Therefore, on $\mathrm{Au}(111)$ the adsorbates can sit at the optimal adsorption position and still have a nearly perfect moleculemolecule distance along the chain direction. However, along the stacking direction the conceptual molecule-molecule distance of $1.036 \mathrm{~nm}$ is approximately $23 \%$ larger compared to the periodicity along the stacking direction in the bulk structure. The larger molecule periodicity weakens the molecule-molecule interactions between two neighboring chains to a negligible value, which explains the observed single chains (see Figs. 4 and 5).

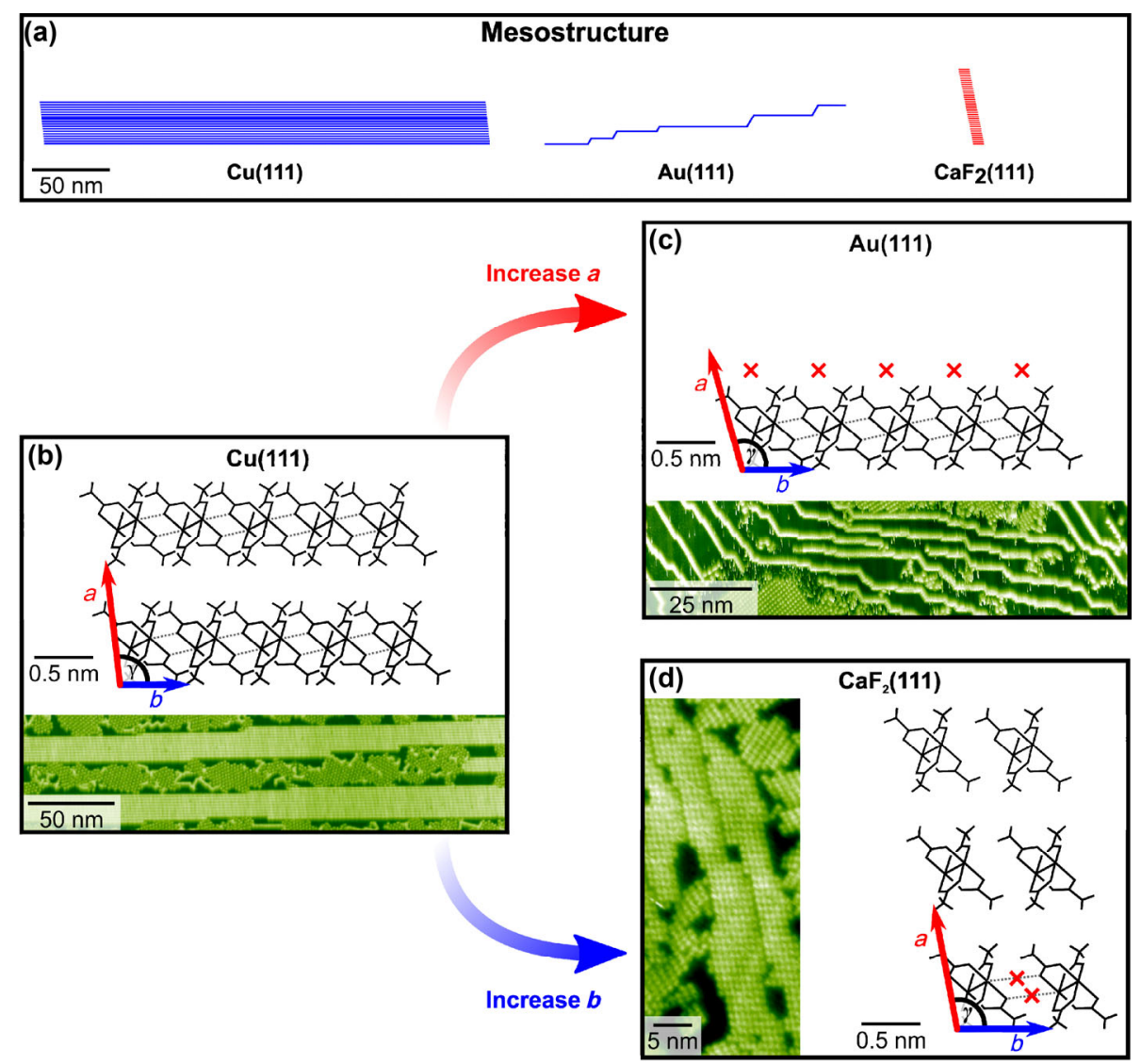

Figure 5 Illustration of mesoscopic structure as a function of the substrates' lattices constant. (a) Comparison of MoMo island shapes on the substrates $\mathrm{Cu}(111), \mathrm{Au}(111)$ and $\mathrm{CaF}_{2}(111)$ at low temperature (110 K and $130 \mathrm{~K}$, respectively). (b) The $\mathrm{Cu}$ (111) lattice allows a favorable molecule-molecule distance along the chain direction (indicated by a blue arrow), but induces a larger molecule distance along the stacking direction (indicated by a red arrow) as compared to the molecular bulk. Consequently, the islands are strongly elongated along the chain direction and short along the stacking direction. Next to the chain islands, there exists a mesh phase, which is not considered here. (c) The slightly larger lattice constant on Au(111) results in a large molecule-molecule distance along the stacking direction. Therefore, only one-dimensional rows, elongated along the chain direction are observed. (d) The periodicity of the $\mathrm{CaF}_{2}(111)$ substrate and site specific adsorption leads to a similar molecule-molecule distance along the stacking direction as compared to $\mathrm{Cu}(111)$, but forces the molecules to increase the distance along the chain direction. In contrast to the island shape on $\mathrm{Cu}(111)$, the islands on $\mathrm{CaF}_{2}(111)$ are, thus, elongated in the stacking direction and have a short chain length. The red crosses in (c) and (d) indicate a weakened molecule-molecule interaction compared to on $\mathrm{Cu}(111)$, caused by a larger molecule spacing along this direction. 
By shedding light on the origin of the observed mesostructure of MoMo on $\mathrm{Cu}(111)$ and $\mathrm{Au}(111)$, we successfully demonstrated the presented strategy to control the self-assembled mesostructure by tuning the lattice constant. Changing the substrates from $\mathrm{Cu}(111)$ to $\mathrm{Au}(111)$ transforms two-dimensional islands into one-dimensional rows (see Figs. 5(a)-5(c)). Furthermore, we gain insights into the range of influence of the moleculemolecule interactions. While a molecule distance of $1.020 \mathrm{~nm}$ on $\mathrm{Cu}(111)$-being equal to a mismatch of $0.178 \mathrm{~nm}$ compared to the ideal bulk configuration-still allows a weak interaction between neighboring chains, a slightly larger mismatch of $0.194 \mathrm{~nm}$ on $\mathrm{Au}(111)$ already results in a negligible moleculemolecule interaction.

As shown above, the described method to control the mesostructure by varying the lattice constant has worked well for the metallic substrates $\mathrm{Cu}(111)$ and $\mathrm{Au}(111)$, which exhibit a similar electronic structure. In the following, we will critically discuss to which extend this method can be transferred to bulk insulators like $\mathrm{CaF}_{2}(111)$ and compare our prediction to the observed self-assembled MoMo island structures.

Interestingly, MoMo on $\mathrm{CaF}_{2}(111)$ exhibits site specific adsorption and, hence, a commensurate superstructure as on the noble metal surfaces and a similar molecule orientation of nearly upright standing molecules in the chain phase. However, it reveals a completely different mesoscopic island shape. On the insulator, the distance between two chains along the stacking direction is equal to the one on $\mathrm{Cu}(111)$, about $20 \%$ larger than the bulk value. However, the molecule spacing along the chain direction is approximately $40 \%$ larger as compared to the bulk structure (see Table 1). Therefore, on $\mathrm{CaF}_{2}(111)$, the molecule-molecule interaction along the chains is expected to be greatly reduced. Consequently, on $\mathrm{CaF}_{2}(111)$ one expects the island growth to be suppressed along the chain direction. As predicted, the islands on $\mathrm{CaF}_{2}(111)$ are predominately elongated along the stacking direction, but with a modest length $(\sim 40 \mathrm{~nm})$ as compared to the island length on $\mathrm{Cu}(111)$ $(\sim 300 \mathrm{~nm})$ as illustrated in Fig. 5(d). Furthermore, at low temperature, the islands are considerably larger on $\mathrm{Cu}(111)$ as compared to $\mathrm{CaF}_{2}(111)$ (see Fig. 5(b)).

Regardless of the substrates microscopic electronic differences, the commensurate superstructure and the similar moleculemolecule interaction mechanism of MoMo on $\mathrm{Cu}(111), \mathrm{Au}(111)$ and $\mathrm{CaF}_{2}(111)$ enables the possibility to successfully control the islands mesostructure by changing the substrates' lattice constant. For explaining the resulting mesoscopic island structure, we make use of strong simplifications. Therefore, we understand that systems can exist where this simple model will break down. However, for the three examples tested here, our simple model provides a reasonable explanation for the resulting mesoscopic island shapes.

\section{Conclusion}

A strategy is presented for tuning the mesoscopic island shape by rationally changing the underlying substrates' lattice constant. As a model system, MoMo molecules are deposited on three different surfaces, $\mathrm{Cu}(111), \mathrm{Au}(111)$ and $\mathrm{CaF}_{2}(111)$. The used substrates are similar as they exhibit hexagonal symmetry, but, compared to $\mathrm{Cu}(111), \mathrm{Au}(111)$ and $\mathrm{CaF}_{2}(111)$ offer by a factor of 1.1 and 1.5 larger lattice constants, respectively. Our scanning probe microscopy results reveal elongated islands in all cases, however, the internal molecule stacking and the directions with respect to the orientation of the substrate differ significantly. For $\mathrm{Cu}(111)$, two-dimensional islands are elongated along the chain direction while for $\mathrm{CaF}_{2}(111)$, two-dimensional islands are elongated along the stacking direction. For $\mathrm{Au}(111)$, in contrast to the other surfaces, only one-dimensional single rows along the chain direction are formed. These differences in the mesoscopic shape can be rationalized by the subtle balance between molecule-surface and intermolecular interactions at the molecular level. The commensurability of the structures dictates the intermolecular spacing in the islands and, thus, the thermodynamically most favorable island shape is a direct consequence of the lattice mismatch. For $\mathrm{Cu}(111)$, we conclude that the lattice dimension fits perfectly to the chain direction of the molecular bulk structure, while in the stacking direction the substrate induces a $20 \%$ increased moleculemolecule distance. As predicted, we found for $\mathrm{Au}(111)$ a slightly larger mismatch of $23 \%$ along the stacking direction, weakening the interaction between neighboring chains even further to a negligible value. Hence, for $\mathrm{Au}(111)$ only single rows form along the chain direction. For $\mathrm{CaF}_{2}(111)$, the moleculemolecule distance is extended in both directions, however, in this case the stacking direction is more favorable as it exhibits the smaller deviation (both absolute and percentagewise). Interestingly, for site specific adsorption the mesoscopic island shape can be rationalized from the surface geometry alone, without considering microscopic electronic details. This work contributes to a comprehensive understanding of tuning mesoscopic island shapes by controlling the interactions at the molecular scale, which is mandatory for understanding the performance of future molecular devices.

\section{Author contributions}

The manuscript was written through contributions of all authors. All authors have given approval to the final version of the manuscript.

\section{Acknowledgements}

S. A. is a recipient of a DFG-fellowship through the Excellence Initiative by the Graduate School Materials Science in Mainz (GSC 266). L. L., B. S., and M. A. thank the Deutsche Forschungsgemeinschaft (DFG, SFB/TRR 88 "Cooperative Effects in Homo- and Heterometallic Complexes (3MET)" Project C5) for financial support and Markus Gerhards (University of Kaiserslautern) for stimulating discussions.

Funding note Open access funding provided by Projekt DEAL.

Open Access This article is licensed under a Creative Commons Attribution 4.0 International License, which permits use, sharing, adaptation, distribution and reproduction in any medium or format, as long as you give appropriate credit to the original author(s) and the source, provide a link to the Creative Commons licence, and indicate if changes were made.

The images or other third party material in this article are included in the article's Creative Commons licence, unless indicated otherwise in a credit line to the material. If material is not included in the article's Creative Commons licence and your intended use is not permitted by statutory regulation or exceeds the permitted use, you will need to obtain permission directly from the copyright holder.

To view a copy of this licence, visit http://creativecommons.org/licenses/by/4.0/.

\section{References}

[1] Lehn, J. M. Supramolecular chemistry-scope and perspectives molecules, supermolecules, and molecular devices (Nobel Lecture). Angew. Chem., Int. Ed. 1988, 27, 89-112. 
[2] Barth, J. V.; Costantini, G.; Kern, K. Engineering atomic and molecular nanostructures at surfaces. Nature 2005, 437, 671-679.

[3] Barth, J. V. Molecular architectonic on metal surfaces. Annu. Rev. Phys. Chem. 2007, 58, 375-407.

[4] Erler, P.; Schmitt, P.; Barth, N.; Irmler, A.; Bouvron, S.; Huhn, T.; Groth, U.; Pauly, F.; Gragnaniello, L.; Fonin, M. Highly ordered surface self-assembly of $\mathrm{Fe}_{4}$ single molecule magnets. Nano Lett. 2015, 15, 4546-4552.

[5] Leonhardt, E. J.; Van Raden, J. M.; Miller, D.; Zakharov, L. N.; Alemán, B.; Jasti, R. A bottom-up approach to solution-processed, atomically precise graphitic cylinders on graphite. Nano Lett. 2018, 18, 7991-7997.

[6] Timmer, A.; Mönig, H.; Uphoff, M.; Díaz Arado, O.; Amirjalayer, S.; Fuchs, H. Site-specific adsorption of aromatic molecules on a metal/metal oxide phase boundary. Nano Lett. 2018, 18, 4123-4129.

[7] Whitesides, G. M.; Mathias, J. P.; Seto, C. T. Molecular self-assembly and nanochemistry: A chemical strategy for the synthesis of nanostructures. Science 1991, 254, 1312-1319.

[8] Nieckarz, D.; Rżysko, W.; Szabelski, P. On-surface self-assembly of tetratopic molecular building blocks. Phys. Chem. Chem. Phys. 2018, 20, 23363-23377.

[9] De Feyter, S.; De Schryver, F. C. Two-dimensional supramolecular self-assembly probed by scanning tunneling microscopy. Chem. Soc. Rev. 2003, 32, 139-150.

[10] Bauer, O.; Mercurio, G.; Willenbockel, M.; Reckien, W.; Schmitz, C. H.; Fiedler, B.; Soubatch, S.; Bredow, T.; Tautz, F. S.; Sokolowski, M. Role of functional groups in surface bonding of planar $\pi$-conjugated molecules. Phys. Rev. B 2012, 86, 235431.

[11] Willenbockel, M.; Lüftner, D.; Stadtmüller, B.; Koller, G.; Kumpf, C.; Soubatch, S.; Puschnig, P.; Ramsey, M. G.; Tautz, F. S. The interplay between interface structure, energy level alignment and chemical bonding strength at organic-metal interfaces. Phys. Chem. Chem. Phys. 2015, 17, 1530-1548.

[12] Stepanow, S.; Ohmann, R.; Leroy, F.; Lin, N.; Strunskus, T.; Wöll, C.; Kern, K. Rational design of two-dimensional nanoscale networks by electrostatic interactions at surfaces. ACS Nano 2010, 4, 1813-1820.

[13] Zhao, W. H.; Zhu, H.; Song, H. J.; Liu, J.; Chen, Q. W.; Wang, Y.; $\mathrm{Wu}, \mathrm{K}$. Adsorption and assembly of photoelectronic TioPc molecules on coinage metal surfaces. J. Phys. Chem. C 2018, 122, 7695-7701.

[14] Huempfner, T.; Sojka, F.; Forker, R.; Fritz, T. Growth of coronene on (100)- and (111)-surfaces of fcc-crystals. Surf. Sci. 2015, 639, 80-88.

[15] Mannsfeld, S. C. B.; Fritz, T. Understanding organic-inorganic heteroepitaxial growth of molecules on crystalline substrates: Experiment and theory. Phys. Rev. B 2005, 71, 235405.

[16] Kumar, A.; Banerjee, K.; Liljeroth, P. Molecular assembly on twodimensional materials. Nanotechnology 2017, 28, 082001.

[17] He, X. Y.; Zhang, L.; Chua, R.; Wong, P. K. J.; Arramel, A.; Feng, Y. P.; Wang, S. J.; Chi, D. Z.; Yang, M.; Huang, Y. L. et al. Selective self-assembly of 2,3-diaminophenazine molecules on $\mathrm{MoSe}_{2}$ mirror twin boundaries. Nat. Comm. 2019, 10, 2847.

[18] Lazarov, V. K.; Cai, Z. H.; Yoshida, K.; Zhang, K. H. L.; Weinert, M.; Ziemer, K. S.; Hasnip, P. J. Dynamically stabilized growth of polar oxides: The case of $\mathrm{MgO}(111)$. Phys. Rev. Lett. 2011, 107, 056101.

[19] Goniakowski, J.; Finocchi, F.; Noguera, C. Polarity of oxide surfaces and nanostructures. Rep. Prog. Phys. 2008, 71, 016501.

[20] Noguera, C. Polar oxide surfaces. J. Phys.: Condens. Matter 2000, 12, R367-R410.

[21] White, T. W.; Martsinovich, N.; Troisi, A.; Costantini, G. Quantifying the "subtle interplay" between intermolecular and molecule-substrate interactions in molecular assembly on surfaces. J. Phys. Chem. C 2018, 122, 17954-17962.

[22] Otero, R.; Gallego, J. M.; De Parga, A. L. V.; Martín, N.; Miranda, R. Molecular self-assembly at solid surfaces. Adv. Mater. 2011, 23, $5148-5176$.

[23] Slater, A. G.; Beton, P. H.; Champness, N. R. Two-dimensional supramolecular chemistry on surfaces. Chem. Sci. 2011, 2, 14401448 .

[24] Goronzy, D. P.; Ebrahimi, M.; Rosei, F.; Arramel; Fang, Y.; De Feyter, S.; Tait, S. L.; Wang, C.; Beton, P. H.; Wee, A. T. S. et al. Supramolecular assemblies on surfaces: Nanopatterning, functionality, and reactivity. ACS Nano 2018, 12, 7445-7481.

[25] Cun, H. Y.; Wang, Y. L.; Du, S. X.; Zhang, L.; Zhang, L. Z.; Yang, B.;
He, X. B.; Wang, Y.; Zhu, X. Y.; Yuan, Q. Z. et al. Tuning structural and mechanical properties of two-dimensional molecular crystals: The roles of carbon side chains. Nano Lett. 2012, 12, 1229-1234.

[26] Gesquière, A.; Jonkheijm, P.; Hoeben, F. J. M.; Schenning, A. P. H. J.; De Feyter, S.; De Schryver, F. C.; Meijer, E. W. 2D-structures of quadruple hydrogen bonded oligo(p-phenylenevinylene)s on graphite: Self-assembly behavior and expression of chirality. Nano Lett. 2004, 4, 1175-1179.

[27] Mukherjee, A.; Sanz-Matias, A.; Velpula, G.; Waghray, D.; Ivasenko, O.; Bilbao, N.; Harvey, J. N.; Mali, K. S.; De Feyter, S. Halogenated building blocks for $2 \mathrm{D}$ crystal engineering on solid surfaces: Lessons from hydrogen bonding. Chem. Sci. 2019, 10, 3881-3891.

[28] Kühnle, A.; Molina, L. M.; Linderoth, T. R.; Hammer, B.; Besenbacher, F. Growth of unidirectional molecular rows of cysteine on $\mathrm{Au}(110)$ $(1 \times 2)$ driven by adsorbate-induced surface rearrangements. Phys. Rev. Lett. 2004, 93, 086101.

[29] Rahe, P.; Nimmrich, M.; Greuling, A.; Schütte, J.; Stará, I. G.; Rybáček, J.; Huerta-Angeles, G.; Starý, I.; Rohlfing, M.; Kühnle, A. Toward molecular nanowires self-assembled on an insulating substrate: Heptahelicene-2-carboxylic acid on calcite (1014). J. Phys. Chem. C 2010, 114, 1547-1552.

[30] Pawin, G.; Wong, K. L.; Kwon, K. Y.; Bartels, L. A homomolecular porous network at a $\mathrm{Cu}(111)$ surface. Science 2006, 313, 961-962.

[31] Li, J.; Wieghold, S.; Öner, M. A.; Simon, P.; Hauf, M. V.; Margapoti, E.; Garrido, J. A.; Esch, F.; Palma, C. A.; Barth, J. V. Three-dimensional bicomponent supramolecular nanoporous self-assembly on a hybrid all-carbon atomically flat and transparent platform. Nano Lett. 2014, 14, 4486-4492.

[32] Kim, H. W.; Jung, J.; Han, M.; Ku, J.; Kuk, Y.; Kim, Y. Dimensionality control of self-assembled azobenzene derivatives on a gold surface. J. Phys. Chem. C 2019, 123, 8859-8864.

[33] Kezilebieke, S.; Amokrane, A.; Boero, M.; Clair, S.; Abel, M.; Bucher, J. P. Steric and electronic selectivity in the synthesis of Fe-1,2,4,5-tetracyanobenzene (TCNB) complexes on Au(111): From topological confinement to bond formation. Nano Res. 2014, 7, 888-897.

[34] Keeling, D. L.; Oxtoby, N. S.; Wilson, C.; Humphry, M. J.; Champness, N. R.; Beton, P. H. Assembly and processing of hydrogen bond induced supramolecular nanostructures. Nano Lett. 2003, 3, 9-12.

[35] Theobald, J. A.; Oxtoby, N. S.; Phillips, M. A.; Champness, N. R.; Beton, P. H. Controlling molecular deposition and layer structure with supramolecular surface assemblies. Nature 2003, 424, 1029-1031.

[36] Blunt, M. O.; Hu, Y.; Toft, C. W.; Slater, A. G.; Lewis, W.; Champness, N. R. Controlling the two-dimensional self-assembly of functionalized porphyrins via adenine-thymine quartet formation. J. Phys. Chem. C 2018, 122, 26070-26079.

[37] Pfeiffer, C. R.; Pearce, N.; Champness, N. R. Complexity of twodimensional self-assembled arrays at surfaces. Chem. Commun. 2017, 53, 11528-11539.

[38] Goiri, E.; Matena, M.; El-Sayed, A.; Lobo-Checa, J.; Borghetti, P.; Rogero, C.; Detlefs, B.; Duvernay, J.; Ortega, J. E.; De Oteyza, D. G. Self-assembly of bicomponent molecular monolayers: Adsorption height changes and their consequences. Phys. Rev. Lett. 2014, 112, 117602.

[39] Stadtmüller, B.; Lüftner, D.; Willenbockel, M.; Reinisch, E. M.; Sueyoshi, T.; Koller, G.; Soubatch, S.; Ramsey, M. G.; Puschnig, P.; Tautz, F. S. et al. Unexpected interplay of bonding height and energy level alignment at heteromolecular hybrid interfaces. Nat. Commun. $\mathbf{2 0 1 4}, 5,3685$.

[40] Hooks, D. E.; Fritz, T.; Ward, M. D. Epitaxy and molecular organization on solid substrates. Adv. Mater. 2001, 13, 227-241.

[41] Zhou, H. T.; Zhang, L. Z.; Mao, J. H.; Li, G.; Zhang, Y.; Wang, Y. L.; Du, S. X.; Hofer, W. A.; Gao, H. J. Template-directed assembly of pentacene molecules on epitaxial graphene on $\mathrm{Ru}(0001)$. Nano Res. 2013, 6, 131-137.

[42] Kalashnyk, N.; Ledieu, J.; Gaudry, É.; Cui, C.; Tsai, A. P.; Fournée, V. Building 2D quasicrystals from 5-fold symmetric corannulene molecules. Nano Res. 2018, 11, 2129-2138.

[43] Cotton, F. A.; Daniels, L. M.; Hillard, E. A.; Murillo, C. A. The lengths of molybdenum to molybdenum quadruple bonds: Correlations, explanations, and corrections. Inorg. Chem. 2002, 41, 2466-2470. 
[44] Kelley, M. H.; Fink, M. The molecular structure of dimolybdenum tetra-acetate. J. Chem. Phys. 1982, 76, 1407-1416.

[45] Ross, R. G.; Hume-Rothery, W. High temperature X-ray metallography: I. A new debye-scherrer camera for use at very high temperatures II. A new parafocusing camera III. Applications to the study of chromium, hafnium, molybdenum, rhodium, ruthenium and tungsten. J. Less Common Met. 1963, 5, 258-270.

[46] Lawton, D.; Mason, R. The molecular structure of molybdenum(II) acetate. J. Am. Chem. Soc. 1965, 87, 921-922.

[47] Cotton, F. A.; Mester, Z. C.; Webb, T. R. Dimolybdenum tetraacetate. Acta Crystallogr. Sect. B: Struct. Sci., Cryst. Eng. Mater. 1974, 30, 2768-2770

[48] Hino, K.; Saito, Y.; Benard, M. Electron-density distribution in crystals of tetra- $\mu$-acetato-dimolybdenum(Mo-Mo). Acta Crystallogr. Sect. B: Struct. Sci., Cryst. Eng. Mater. 1981, 37, 2164-2170.

[49] Lichtenberger, D. L.; Ray, C. D.; Stepniak, F.; Chen, Y.; Weaver, J. H. The electronic nature of the metal-metal quadruple bond: Variable photon energy photoelectron spectroscopy of $\mathrm{Mo}_{2}\left(\mathrm{O}_{2} \mathrm{CCH}_{3}\right)_{4}$. J. Am. Chem. Soc. 1992, 114, 10492-10497.

[50] Lichtenberger, D. L.; Kristofzski, J. G. Intermolecular influences on M-M multiple bonds from thin-film UPS studies of group VI $\mathrm{M}_{2}\left(\mathrm{O}_{2} \mathrm{CCH}_{3}\right)_{4}$ complexes. J. Am. Chem. Soc. 1987, 109, 3458-3459.

[51] Engelhardt, J. B.; Dabringhaus, H.; Wandelt, K. Atomic force microscopy study of the $\mathrm{CaF}_{2}(111)$ surface: From cleavage via island to evaporation topographies. Surf. Sci. 2000, 448, 187-200.

[52] Albrecht, T. R.; Grütter, P.; Horne, D.; Rugar, D. Frequency modulation detection using high- $Q$ cantilevers for enhanced force microscope sensitivity. J. Appl. Phys. 1991, 69, 668-673.

[53] Kollamana, J.; Wei, Z.; Lyu, L.; Zimmer, M.; Dietrich, F.; Eul, T.; Stöckl, J.; Maniraj, M.; Ponzoni, S.; Cinchetti, M. et al. Control of cooperativity through a reversible structural phase transition in momo-methyl/Cu(111). Adv. Funct. Mater. 2018, 28, 1703544.

[54] Foster, A. S.; Barth, C.; Shluger, A. L.; Reichling, M. Unambiguous interpretation of atomically resolved force microscopy images of an insulator. Phys. Rev. Lett. 2001, 86, 2373-2376.

[55] Foster, A. S.; Barth, C.; Shluger, A. L.; Nieminen, R. M.; Reichling, $\mathrm{M}$. Role of tip structure and surface relaxation in atomic resolution dynamic force microscopy: $\mathrm{CaF}_{2}(111)$ as a reference surface. Phys. Rev. B 2002, 66, 235417. 